\title{
Complex Systems Engineering Approach for Condition Monitoring for the Digital Transformation: Integration into Mining Industry Control Systems
}

\author{
Mariya Guerroum $^{1,2(\bowtie)}$, Ali El-Alaoui ${ }^{1,3}$, Laurent Deshayes ${ }^{1}$, \\ Mourad Zegrari ${ }^{1,2}$, Janah Saadi ${ }^{3}$, and Hicham Medromi ${ }^{3}$ \\ ${ }^{1}$ Innovation Lab for Operations, Mohammed VI Polytechnic University, \\ Ben Guerir, Morocco \\ \{Mariya.guerroum, ali.elalaoui\}@um6p.ma \\ ${ }^{2}$ Ecole Nationale Supérieure des Arts et Métiers de Casablanca, \\ Casablanca, Morocco \\ ${ }^{3}$ Ecole Nationale Supérieure de l'Electricité et de la Mécanique, \\ Casablanca, Morocco
}

\begin{abstract}
The digital transformation of the Mining Industry is about to affect all organizational levels, from manufacturing to maintenance. This revolution would be impossible without a modern Information and Communication Technologies (ICT) infrastructure. Maintenance management is a complex process requiring an effective combination of technical and economic expertise. This paper presents electromechanical systems condition-monitoring architecture, using the Systems Engineering Approach. The main function of the system is to extract and to identify physical parameters of the studied system for predictive maintenance strategy elaboration. The System's architecture based on the operational, functional and constructional visions is fundamental to define the action scope and its features, leading to avoid production breakdowns, to improve maintenance management and to minimize the related intervention costs. We used SysML diagrams to model the solution and hence to materialize the System targeting maintenance in industrial environment.
\end{abstract}

